\title{
Discurso Pedagógico y Subjetivación
}

Recibido: 14.08.2020 / Aprobado: 20.10.2020

\section{RESUMEN}

Este texto forma parte de una investigación que reflexiona sobre las potencialidades y oportunidades de la formación responsiva, se inscribe en el tiempo y contexto del ingreso del nuevo estudiante a la vida universitaria, en parte por las mediaciones y discursos situados que procura leer, así como la propia distancia que guardo con el recurso memorístico para esta tarea, ya que comparto con Braunstein (2008) que la memoria negocia el recuerdo a base de amabilizaciones.

El desarrollo de esta tarea se agenció a través de la reflexividad comparativa, partiendo de la hipótesis que la vida y reproducción del currículo y su discurso pedagógico comprometen la agencialidad de sus agentes, a través de acciones y relaciones ideológicamente tensas en lo simbólico. Con ello se pretende tributar tanto a la reflexión como a la concreción pedagógica de estrategias afirmativas a la agencia, -como capacidades para actuar $\mathrm{y}$ transformar-. Entre los hallazgos más relevantes de esta tarea están: Una creciente desconfianza en nociones funcionalistas que aíslan el sesgo de lo oculto de la propia práctica real del currículo. Asimismo, la comprobación de las implicancias reales de las acciones y relaciones tensas desde lo simbólico -violencia simbólica, racismo, sexismo y clase- .

Palabras clave: Agencia, sujeto, otro, discurso pedagógico, violencia simbólica.

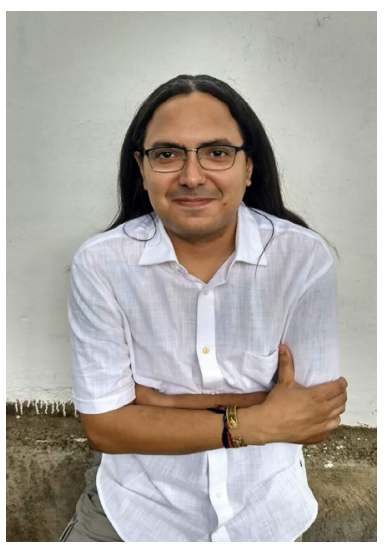

Por Ixbalanqué Ocón

Master en Ciencias Sociales. Universidad Politécnica de Nicaragua. Correos: ieg-docente2@upoli.edu.ni ixbalanque1@gmail.com.
Por Ixbalanqué Ocón
This text is part of an investigation that reflects on the potentialities and opportunities of responsive training. It is inscribed in the time and context of the new student's entry into university life, in part due to the mediations and situated discourses that he tries to read as well as the own distance that I keep with the memory resource for this task, since I share with Braunstein (2008) that the memory negotiates memory based on kindnesses.

The development of this task was organized through comparative reflexivity, starting from the hypothesis that the life and reproduction of the curriculum and its pedagogical discourse compromise the agency of its agents, through actions and ideologically tense relations in the symbolic. This is intended to contribute both to reflection and to the pedagogical concretion of affirmative strategies to the agency, -as capacities to act and transform-. Among the most relevant findings of this task are: A growing distrust in functionalist notions that isolate the bias of the hidden from the actual practice of the curriculum itself. Also, the verification of the real implications of tense actions and relationships from the symbolic point of view - symbolic violence, racism, sexism and class. $^{1}$

Key Words: agency, subject, other, pedagogical discourse, symbolic violence.

https://orcid.org/0000-0001-9489-1585

Traduacción del Autor 


\section{Introducción}

El presente informe es un intento de registro reflexivo sobre la práctica del discurso pedagógico, a través de un ejercicio de observación directamente a un grupo de primer año de una carrera universitaria, así como visitas a otro grupo, a modo comparativo, atendiendo a la especificidad de las relaciones en lo tácito del discurso, consciente de las limitaciones implícitas a la posición de quien investiga con fines y a priori.

Decidí tomar decisiones mediadas por mi propia intuición y subjetividad, algunas de estas fueron: Las carreras, las cuales desde la vida del currículo me representan por sus campos y fines mayores posibilidades y oportunidades para el desarrollo de pedagogías sensibles al yo, es decir al desarrollo de la agencia personal del estudiante en torno al desarrollo de sus capacidades de aprendizaje y ejercicio del poder.

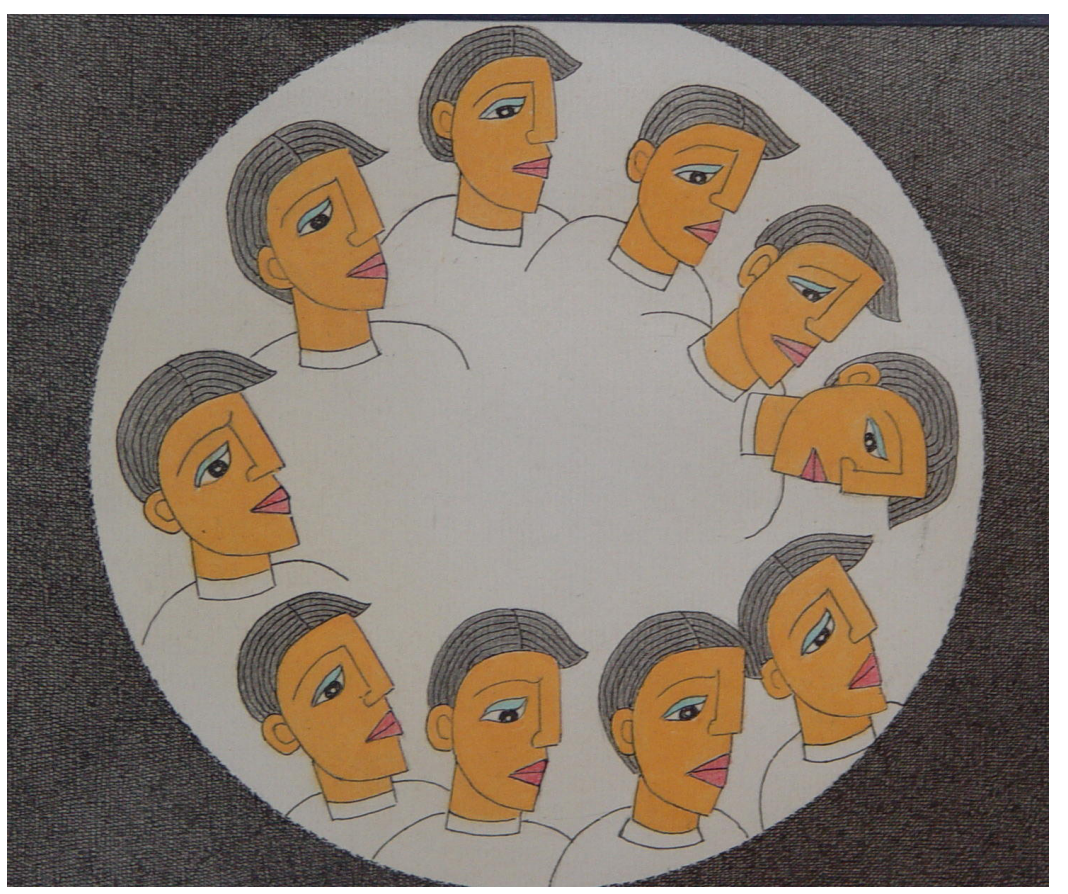

Detalle de obra de arte del Maestro Leoncio Sáenz

\section{Pre-ocupaciones teóricas, metodológicas y éticas}

Parto del supuesto y condición clave para este análisis, sobre la idoneidad del momento y contexto; del ingreso a la vida universitaria, ya que por un lado, esto me aproxima a sujetos descontextualizados y des-familiarizados del discurso pedagógico institucional. Desde lo personal y sobre la base de mi experiencia práctica y teórica como docente de estudiantes universitarios de nuevo ingreso, encuentro fructífero este momento para

el análisis, dada las mediaciones y localizaciones de los discursos.

Ello en parte, guarda relación al hecho de que como investigador no disimule mis c o m p r o m e t i d o s presupuestos con una mirada socio crítica, donde por ejemplo, me muestro afín a la idea de la relación sinécdoque que guardan los sujetos con sus contextos a través de tradicionales mediaciones McLaren (1997), de manera que esta tarea me supone la emergencia de algunas de

De esta manera, intuyendo de sus discursos pedagógicos -además por la naturaleza de sus campos discursivosterrenos ideológicos fructíferos para la finalidad de la investigación a la que esta tarea responde, la cual persigue la identificación y análisis de condiciones objetivas y subjetivas que potencien la emergencia y desarrollo de esta voz propia que supone la agencialidad; como una respuesta agonista a la reinante opacidad del estudiantado universitario, que exhibe no sólo una relación epocal, sino antagónica para con los fines y principios del acto pedagógico. Ello en el marco de una institución de educación superior privada de servicio público, cuyos discursos pedagógicos se presentan antinómicos frente al currículo prescrito. las tensiones que intento comprender, no sólo por parte del nuevo estudiantado, sino de los propios docentes, esta pretensión de presenciar lo más cerca posible que el grupo me lo permita- está atravesada por mis experiencias, teorías y posicionamientos.

Elegí este emplazamiento sumamente convencido del enorme vacío, siquiera descriptivo, amén explicativo aún para este ámbito y momento específico de la educación superior y su impacto en la relación pedagógica, de manera que esta tarea se aventura por auscultar la complejidad de tales mediaciones, ya que en parte el discurso pedagógico se re-reproduce en silencio colectivo, en fin es un tiempo y proceso que no 
ha sido lo suficientemente estudiado, al menos en este contexto.

He de reconocer también serias limitaciones del proceso; como esa contingente subjetivizacion y sus riesgos de verse en el Otro, lo cual constantemente me obligo a mantener una postura de incompletud sobre lo registrado y no apelar a lo dado. Para ello fue clave revisitar con los propios individuos lo acontecido. Ello no sólo actuó a modo de validación comunicativa, sino que me permitió identificar aspectos inadvertidos que cobraron relevancia para los propios sujetos, como su propia valoración de los mismos hechos, "en la búsqueda de sus lenguajes".

Este proceso se llevó a cabo a través de la técnica del análisis de las conversaciones con los propios sujetos -docentes y estudiantes- ya que si bien Sacks (1984) fundador de esta técnica, rescata el valor de la palabra como productora de lo contingente de la producción social y cultural. Ello es también algo profusamente clave en el cuerpo teórico del psicoanálisis. Un cuerpo teórico de gran interés para mí como persona e investigador y es propio Jaques Lacan (1958), quien precisamente insiste en la propiedad de extimidad del inconsciente desde lo hablado.

Consecuentemente, ello supuso emergentes adecuaciones desde lo metodológico, pero también desde lo teórico. Y dado que la investigación no sólo se trata de aprehender el objeto sino también su proceso, cabe resaltar que llegué a identificar que en la práctica investigativa que la teoría no solamente está ahí, sino que no es posible prescindir de ella.

Asimismo, es importante resaltar que en tal proceso de adecuación metodológica a modo de soliloquio ético me orientaron las exhortaciones de Rapley (2014): “[ ...] conozca las posibles implicaciones de sus acciones y sea sensible a ellas" (p.57).Y Bourdieu (2010): “[...] ningún contrato esta tan cargado de exigencias tácitas como un contrato de confianza" (p.7).

\section{Leer para reescribir el discurso (en alusión a la metáfora de Steiner)}

La primera clase se inaugura con un discurso autorreferencial por parte del docente y con ello localiza las distancias entre éste y sus estudiantes, transmitido desde la propia exaltación de sus cualicalidades personales por vías de la confesión narcisista, de la auto-percibida trayectoria de éxito profesional y académico - evidenciando un proceso de elección y producción imaginaria- dejando esto consecuencias irreversibles, como la inscripción de la falta en el Otro -estudiante- o su localización tacita, actuando a modo de brecha simbólica. Esta primera inscripción resulta esencial para el devenir cotidiano dentro del proceso de aprendizaje, al punto que signa la posición de saberpoder y autoridad del discurso pedagógico, y el valor de cierta obediencia al mismo, como garante de éxito. No obstante, este uso del poder también presenta un reverso, ya que a mediano plazo constituirá un elemento generador de subjetivizacion por identificación a modo de aspiraciones imaginarias autorreferenciales en la propia subjetividad del estudiante.

Un aspecto en apariencia larvado del discurso pedagógico y que no supone mayor intencionalidad más que la de generar una suerte de rapport pudo ser identificada al momento de la presentación de los miembros del grupo. No obstante, desde nuestro escenario intersubjetivo como participantes, admitió entrever alguna tensión desde las auto-adscripciones a una determinada clase social. De este modo en el grupo esta práctica resultó dolorosa para algunos, quienes literalmente se expusieron a toda clase de ataques, unos a otros se mancillaban entre miradas y vocecitas jocosas.

Este infame ritual fue solicitado en más de una ocasión, a cada cual desde distintos escenarios o asignaturas. Dichas presentaciones fueron tornándose escabrosas a medida que algunos docentes solicitaban especificaciones sociodemográficas como: procedencia geográfica, nombre del colegio, y hasta el estatus del mismo -si privado o público-. Y si a ello se le agregan, los comentarios "al margen de todo margen" descalificando la educación pública.

Por todo lo anterior no sorprende que en lo tácito del ritual hubiera una incitación a la violencia simbólica, de ahí que la respuesta inmediata desde los propios estudiantes se manifestara a través de complejas prácticas de sanción y vergüenza, generando "un nosotros" bastante claro en un sector importante del 
grupo, una suerte de identificación in situ frente a aquellos que exhibían mayores ventajas. Al respecto de esta práctica considero que encuentra eco en la ferocidad con la que McLaren (2003) define la cultura del salón como sombría, y propiciadora de competencia y conflicto. Esta estrategia de clase-ficación, pareciera ser un elemento privilegiado de la red de significantes del discurso pedagógico.

Con gran certeza puedo afirmar que las tramas de la violencia simbólica ahí acontecidas y cada vez más aparecidas entre el grupo, tuvieron si no su origen, al menos un aliciente en rituales como este. Asimismo, durante sucedieron estos hechos, los ánimos se fueron caldeando al punto de empezar a aparecer en el discurso alguna negociación frente a las propias realidades, al punto de modificar su procedencia aunando la capital a sus respectivos lugares reportados en claro intento por amortiguar la falta frente al Otro. De este modo fueron escuchándose más frecuentemente los MasayaManagua y Matagalpa-Managua como una "mitigadora acción simbólica”.

Este hallazgo me resultó a la vez que dramático fundamental para determinar el impacto de esta violencia en la subjetivación del grupo, también me permitió identificar el lugar que ocupa la procedencia de clase en la red de significantes del discurso pedagógico. No obstante, desde una práctica inconsciente, casi liberada de toda intencionalidad y posiblemente desde un lugar "emocionalmente silencioso", pero que en lo personal me sojuzga y a la vez genera en mí, un distanciamiento con la idea de separar los currículos real y oculto, pues he llegado a la comprensión de que no hay más que mera mediación localizada del propio discurso pedagógico.
Estalógicadelosunosfrenteaotrosmantuvounatendencia en movimiento conforme transcurrían las semanas, al punto de la emergencia de relevantes fenómenos para el análisis, como la aparición del conflicto con el Otro y su otredad, parte de esa heterogeneidad antes descrita en el grupo implica la presencia de estudiantes afrodecendientes, aunque si bien de diferentes etnias y procedencia geográficas y culturales dentro de la Costa Caribe, quienes conforme transcurrían los días fueran relajando sus defensas -yoicas- expresando lo propio de la masculinidad cultural; al respecto de las prerrogativas $\mathrm{y}$ roles de género en torno a la significación $y$ erotización del cuerpo de las mujeres.

Desde las pautas locales, el conflicto tenía sus días contados para aflorar, ya que en las prácticas cotidianas tanto el profesorado como los propios estudiantes no están exentos de miradas a los cuerpos, pero esta práctica guarda un código que no admite el exceso, lo que en si es problemático para estos jóvenes con altos niveles de descontextualización para quienes conocer la medida de este exceso es sumamente complejo, casi inaccesible y problemático, pues les resta identidad.

Prosiguen los días, se suceden los escenarios, a cada docente le caracteriza un tono, una elección de que corregir y sancionar, así las docentes mujeres atenderán aspectos propios de la ortodoxia y sus maneras prohibitivas durante sus clases; el uso de celulares, la ingesta de comida en el aula, la puntualidad, la vestimenta -erotización de los cuerpos- el uso del lenguaje, y no menos importante la invocación del Reglamento de Régimen Estudiantil, en clara disposición ortopédica. Por cierto, son las únicas que desde el primer día de clase se encargan de brindar con lujo de detalles el sistema de evaluación, aun cuando las expectativas del grupo por la clase y sus nexos tributarios al perfil de la carrera no fueran siquiera 
explorados. Lo que recuerda por mucho que no hay mayor antinomia en el socioconstructivismo educativo que imponer objetivos al margen de los intereses propios de los sujetos aprendientes. Amén del sistema de calificación eminentemente funcionalista que cristaliza la verdadera naturaleza del modelo tendiente al "rendimiento".

De este modo, el hecho que desde el primer día de clases sea abordada la evaluación y sus bemoles técnicos, son señales de la primacía de esta por sobre el aprendizaje, de ahí esa "angustia sumativa", la cual enerva las propias posibilidades de posicionarse como sujetos del conocimiento. Así sus agentes -docentes y estudiantesen clara economía simbólica reducen la complejidad que supone el acto de aprender, anteponiendo la evaluación del contenido al posicionamiento del sujeto que aprende.

Sobre estos modelos de rendimiento y su pragmática Bernstein (2000) explica que: "Se centran en algo que el adquiriente no posee, en la inexistencia, sobre una ausencia, y como consecuencia el lugar y énfasis sobre el texto que debe ser a d q u i r i d o" [... $\quad$ (p.57). Un ejemplo ilustrativo de este énfasis en la falta $y$ de paso de la teoría de los códigos (restringidos y elaborados) de Bernstein se constató en una actividad

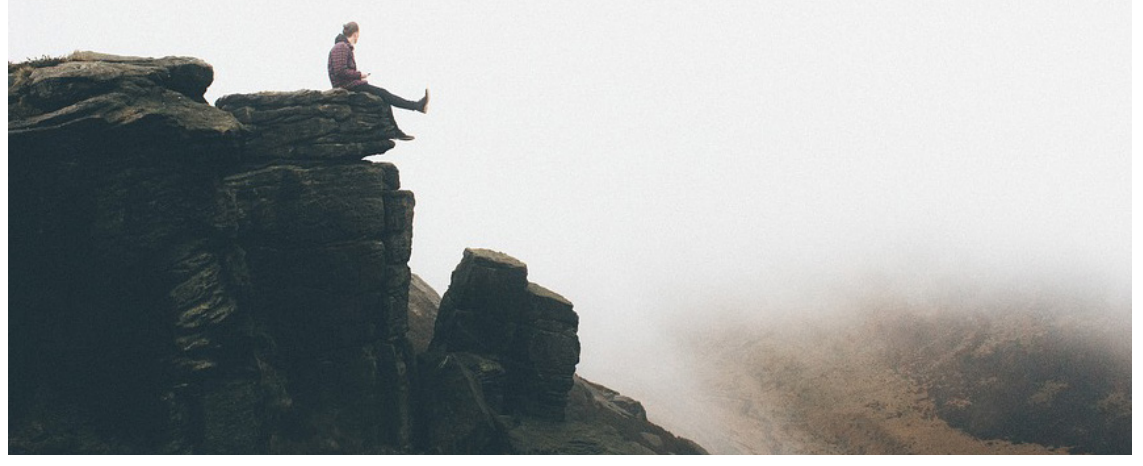

Pixabay.com

categorías, tales como: cero, literal, inferencial y aplicado, hasta el punto de cortar al sexto participante, para desde ahí generar una estadística de los niveles de comprensión del grupo. Ante esta barbarie me sigo preguntando ¿Dicho ejercicio cumplía la función de comunicar algo más que la falta? Yo creo que sí y ello es la propia culpa.

A pesar de este discurso pedagógico hay luces y oportunidades en medio de tanta miseria social para este escenario y es que en medio de tan abrumadores hallazgos logré identificar en escasos dos docentes, prácticas discursivas relativamente disidentes a lo vigente, donde a menor ropaje teórico, lenguaje coloquial, donde la funcionalidad de la risa, si bien hace lo suyo, cumple un propósito positivador el de brindar sentido de grupo alejado de lo necrótico. Asimismo, un cierto tono autoritario - quizás más relajado, menos ortopédico e instrumental- evidenciando una ganancia tendiente más a acuerdos tácitos entre personas.

Para estos docentes en efecto este trabajo desidentificatorio con el discurso vigente les implica riesgos. Riesgos que resulta oportuno tener siempre presentes, al margen de las relaciones pedagógicas. De este modo esta transgresora manera de hacer docencia conscientemente atiende, intuye y se implica. Porque si bien estos encuadres discursivos están escindidos del discurso institucional de corte practicista y ortopédico, no así en sus posibilidades declaradas y oficializadas en las políticas y modelos educativos institucionales de corte socioconstrutivistas, lo que de entrada refuta la tesis de los dos discursos institucionales en la vida del currículo; un antinómico a la propia pedagogía declarada, y su reverso transformador que se muestra sensible a lo dispuesto, que tiende a concretar y extremar sus límites filosóficos. 


\section{Conclusiones}

Aspirar a una formación responsiva afirmativa a la idea de agencialidad brega por la reafirma ción decidida de la autonomía y el cultivo del sí mismo en términos foucaultianos. Pero sin dejar de atender la empatía, ya que como bien plantea Sloterdijk (2014) "Los espíritus regentes se reducen a las dos fuerzas primarias: la erótica, que quiere tomar, y la timótica, que quiere dar" (p.59). Sobre todo, porque la educación como bien perdurable es solo aquella que comprometiendo su teleología promueve intereses propios, o lo que es igual al "inicio de caminos" de sujetos, pero no sólo eso, sino prácticas reflexivas y transformadoras de lo que falta en lo social, es decir contestatarias a la tendencia del deseo individual y la envidia como narrativas axiológicas hegemónicas.

Es ineludible que la práctica del discurso pedagógico presenta serias faltas y que por su carácter sujetado tiende a elegir prioridades, a veces consciente -otras no tanto-, pero en lo general se tiende al conservadurismo de la práctica, nunca a su resignificación, amén de su contestación. Porque como bien nos exhorta Wittgenstein (1947) citado en Steiner (2012):“[ ...] siempre olvidamos descender hasta los fundamentos. No ponemos los signos de interrogación a suficiente profundidad" (p.32).

En definitiva el currículo está vivo, sus docentes y estudiantes lo confirman, el aula ha ampliado sus coordenadas y tiempos el aprendizaje. Hoy día ya no le cabe a la educación la idea bancaria Freire (2005) las potencialidades y oportunidades para construir saberes e intereses, que puedan producir destinos significativos en sus agentes están dadas. Es tiempo ahora de reflexionar y el primero en ser llamado es el docente quien hoy día le caracteriza una acrítica y cristalizada posición imaginaria reproductora de pírricas expectativas sobre su propia agencia y la de su estudiantado.

Recordando que los docentes que se abstienen de declarar su posición cometen el mismo error de aquellos que abiertamente la imponen a sus estudiantes. Tal falta es su "imposibilidad del ejercicio de la reflexibilidad en tanto práctica autocrítica". Porque cuando se es consciente de que cada aula es en parte el mundo social y además, se posibilita tal consciencia en los otros, la ex- presa y demasiada humanidad del mundo social, con toda su miseria y violencia simbólica, más que inadvertida sea invertida, quedando así expuesta sus lógicas, generando condiciones para su contestación.

\section{REFERENCIAS BIBLIOGRÁFICAS}

Augé, M (2000) Los "no lugares" espacios del anonimato: Una antropología de la sobremodernidad. España. Gedisa Editorial.

Bernstein, B. (2000). Pedagogía, control simbólico e identidad: Teoría, investigación y crítica. Madrid: Morata.

Bergson, H. (2013). La risa: Ensayo sobre el significado de la comicidad. Buenos Aires: Ediciones Godot.

Braunstein, Nestor. (2008). La memoria, La inventora. México D.F., México: Siglo XXI.

Bourdieu, P. (2010). La miseria del mundo. Buenos Aires, Argentina: Fondo de Cultura Económica.

McLaren, P. (1997). Pedagogía crítica y cultura depredadora, Políticas de oposición en la era posmoderna. Barcelona, Paidós.

McLaren, P. (2003). La escuela como un performance ritual: Hacia una economía política de los símbolos y gestos educativos. México: Siglo XXI Editores, s.a. de c.v.

Rapley, T. (2014). Los análisis de la conversación, del discurso y de documentos en investigación cualitativa: Colección; Investigación Cualitativa. Madrid: EDICIONES MORATA, S.L.

Sloterdijk, P. (2014). Fiscalidad Voluntaria y responsabilidad ciudadana: Aportaciones a un debate filosófico sobre una nueva fundamentación democrática de los impuestos. Madrid: \#Ediciones Siruela

Steiner, G (2012). La poesía del pensamiento: Del helenismo a Celan. Madrid: Ediciones Siruela. 